\title{
Extra-pleural solitary fibrous tumors: a review
}

\author{
Nuno Almeida Costa*, Diogo Fonseca and João Santos
}

\begin{abstract}
Solitary fibrous tumor (SFT) is a rare mesenchymal ubiquitous tumor reported in the pleura and a wide variety of extrapleural locations, most frequently in the orbits and extremities.

Approximately $78-88 \%$ of SFTs are benign and $12-22 \%$ are malignant.

Although tumor characteristics are highly dependent on the location there are unifying features in MR imaging suggesting SFT: well-circumscribed, often lobulated nodular lesion, delayed contrast enhancement and the presence of fibrous contents which are hypointense on T1 and T2-weighted MRI imaging.

There are many SFT differential diagnosis, highly dependent to the tumor location. Due to its rarity the diagnosis of extrapleural SFTs may be challenging. Histopathologic analysis is always required to confirm the diagnosis and to allow the distinction between the benign and malignant forms.

We review pleural and extrapleural SFTs, presenting diagnostic clues, differential diagnosis and prognostic factors.
\end{abstract}

Keywords: Solitary fibrous tumors, SFT, Extra pleural tumor-ubiquitous

\section{Introduction}

\section{Background}

Solitary fibrous tumors (SFTs) are uncommon tumors arising from mesenchymal cells representing less than $2 \%$ of all soft-tissue tumors [1].

SFTs were firstly described in 1931, referring to pleural localization [2], being described later in other extrathoracic localizations. It is now established that SFTs can originate in virtually any site of the body and the incidence of extrapleural SFTs is shown to be higher than of the pleural lesions [3].

The deep soft tissues of the proximal extremities, abdominal cavity, head (orbits) and neck are the most frequently reported extrathoracic locations [4].

The majority are benign, although up to $20 \%$ may be malignant [1].
Regarding genetic background of the disease, different rearrangement of the NAB2-STAT6 gene has been associated to the pathogenesis of SFTs, potentially related to more aggressive behavior [5].

\section{Clinical and work-up features}

SFT has no gender predilection and manifests as an asymptomatic or slow-growing mass in middle-aged adults [4]. Nevertheless, extrathoracic SFTs have been reported to occur slightly more frequently in women than men [6].

Most of the patients with SFTs are asymptomatic at the time of diagnosis, and the majority of these tumors are discovered on routine chest plain films [7].

Extrapleural SFTs related symptoms are generally non-specific or related to mass effect in the majority of reported cases [8].

A minority of these tumors $(<5 \%)$ may present with hypoglycemia related to excessive production of insulinlike growth factor by the tumor, which is more frequent in pelvic and retroperitoneal SFTs - the Doege-Potter syndrome (hypoglycemia secondary to malignant SFTs) [9].

SFT in unusual locations (paraspinal, paramediastinal, intrafissural, abdominal), can pose interpretation problems or, indeed, point towards a different diagnosis.

\footnotetext{
*Correspondence: nunoada@hotmail.com; nunoadac@gmail.com; nuno.a.costa@ipoporto.min-saude.pt

Radiology Department, Instituto Português de Oncologia do Porto de Francisco Gentil, IPO - Porto, Rua Dr. António Bernardino de Almeida, 4200-072 Porto, Portugal
} 


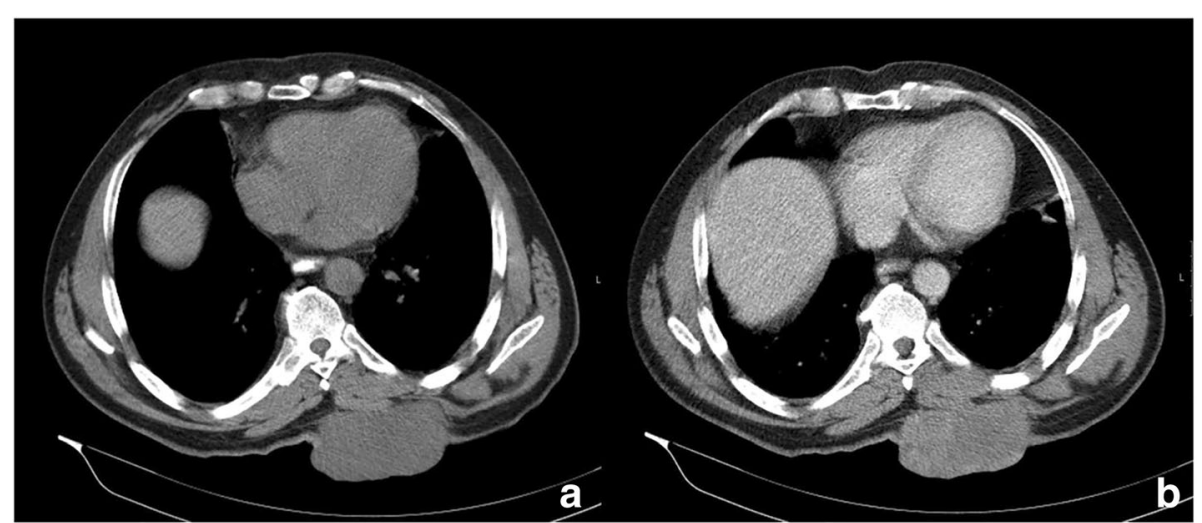

Fig. 1 Dorsal thoracic SFT in man, 55 years. Axial unenhanced (a) and enhanced (b) CT shows a homogeneous lobular enhancing mass in the left posterior thoracic wall with smooth well-defined margins. This patient was submited to resection with no evidence of recurrence to date

The preoperative diagnosis of the tumor is not always possible and the definitive diagnosis requires the histologically evaluation of the resected specimen. Immunochemistry plays a key role in terms of the distinction of SFTs from mesotheliomas and sarcomas [10].

According to the WHO classification SFTs are classified as having intermediate malignant potential with low risk of metastasis [11].

The malignant potential of extrapleural SFTs is similar to pleural tumors, with malignant features detected at pathologic examination in up to $20 \%$ [1]. Thus, most extrapleural SFTs behave in a benign fashion, although there are no sufficient imaging features to predict clinical outcome [4].

Surgical excision is the treatment of choice for all SFTs. The 5 -year survival rate is near $100 \%$ when free margins surgical excision is achieved [12].
SFTs have a low tendency to recur after primary surgery [13] although extrathoracic SFTs show slightly higher local recurrence rates after surgical excision [1].

The treatment of unresectable SFTs is challenging. Small series report conflicting results but overall limited efficacy of chemotherapy [14, 15]. Neoadjuvant radiation therapy has also variable success rates $[16,17]$.

After the surgical treatment of a SFT an extensive follow up is always recommended due to the risk of tumor recurrence [18].

\section{Purpose}

The purpose of this exhibit is to illustrate and describe the spectrum of radiological findings found in extrapleural fibrous solitary tumors, emphasing the CT and MR characteristics. We aim to present a variety of SFT

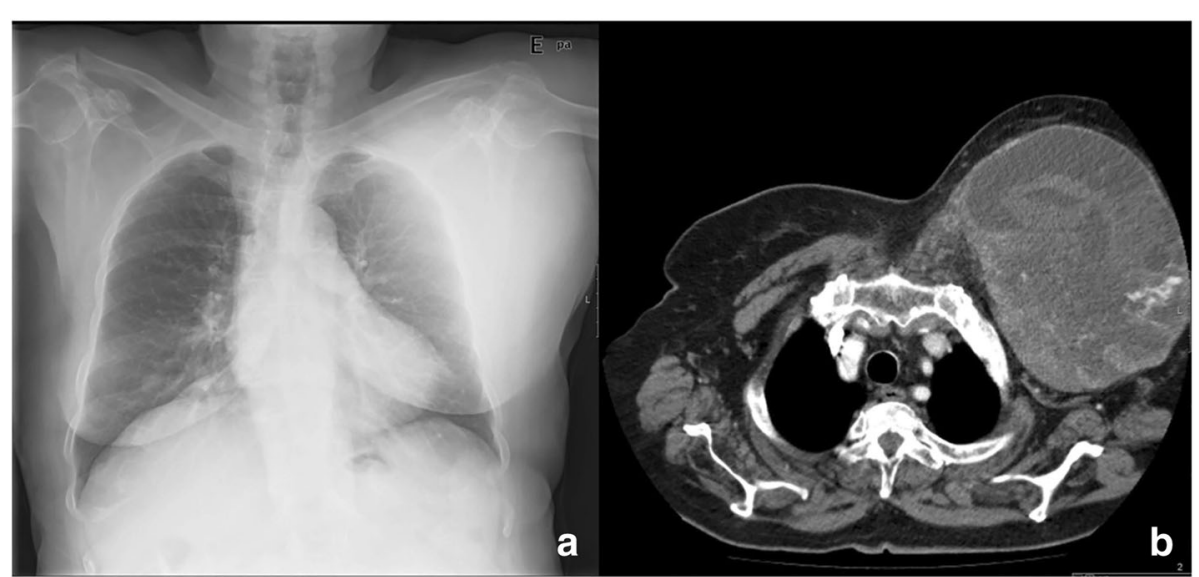

Fig. 2 Thoracic wall SFT. PA radiography (a) and enhanced axial CT images (b) showing a large well-defined exophytic mass with marked heterogenous enhancement. The diagnosis of SFT was obtained by a transthoracic needle biopsy 


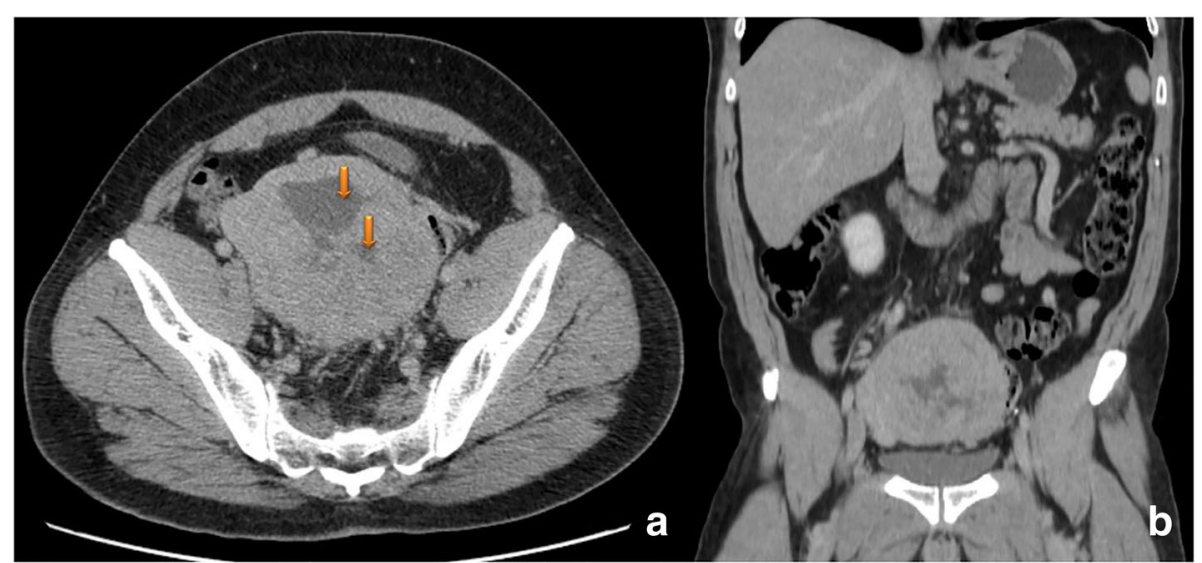

Fig. 3 Typical imaging manifestations of SFT of the peritoneum. Axial (a) and coronal (b) contrast material-enhanced CT scan shows a welldefined intensely enhancing mass in the pelvis with central nonenhancing areas of necrosis (arrows)

including thoracic extra-pleural, SFTs of peritoneum and pelvis, SFT of the limbs and of the neck/paranasal.

\section{Material and methods}

A literature review was performed via a comprehensive electronic search of PubMed, MEDLINE, and Cochrane databases, followed by retrospective analysis of a series of pathologically confirmed extra-pleural SFT of our institution. Tumor characteristics were highlighted, mainly at $\mathrm{CT}$ and MR images.

\section{Theory}

Better understanding of the different patterns of SFTs will probably enhance radiologists ability to suggest this diagnosis and not overlook the extra-pleural variants.

Future statistical analysis of extra-pleural SFTs characteristics, such as the enhancement pattern and the evidence of internal low signal areas on T1 and T2 weighted MR images, should be performed in order to evaluate image criteria for predicting malignant variants.

\section{Results and discussion}

General radiological characteristics

Radiography studies are generally not very helpful in assessing extrapleural SFTs, besides showing internal calcifications in some cases [18].

The ultrasound appearance is non-specific. The limited available ultrasound data states that SFTs are heterogeneous masses with internal vascularity [19].

CT evaluation is generally the first modality imaging study to depict the lesion and is good for defining size, local extent, including invasion into adjacent structures, and detection of regional metastases.

Generally SFTs appear as well-defined, hypervascular mass that often compress adjacent tissues and organs.

Scattered intratumoral foci of hypoenhancement or non-enhancement usually represent regions of necrosis, haemorrhage or cystic change. Nevertheless, smaller lesions typically demonstrate homogeneous enhancement.

Calcifications are uncommon, occurring both in large benign or malignant tumors.

MR evaluation is the preferred modality study for tissue characterization. STFs generally present intermediate

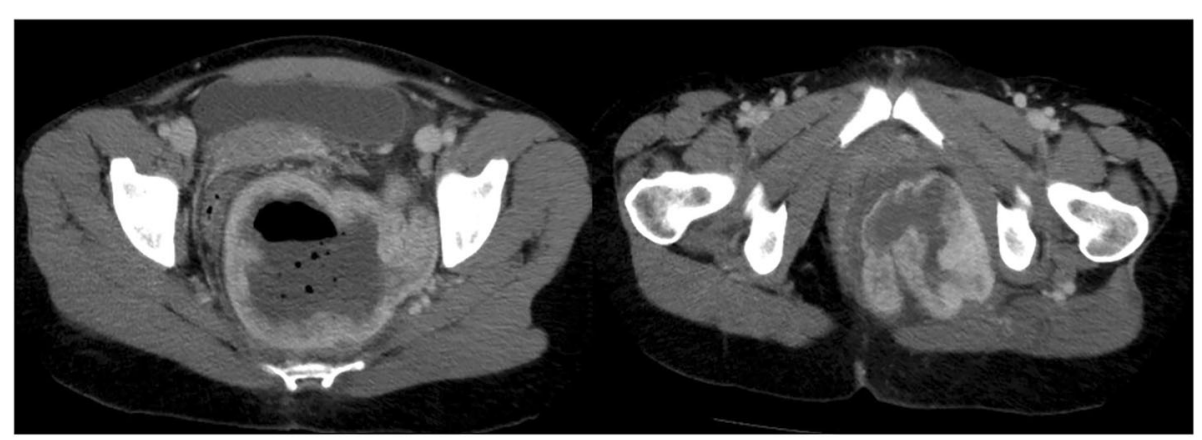

Fig. 4 Atypical presentation of a SFT of the pelvis. Axial CT images showing a large heterogeneous mass with central necrosis and thick walls. It was biopsy proven to be a SFT of the pelvis 


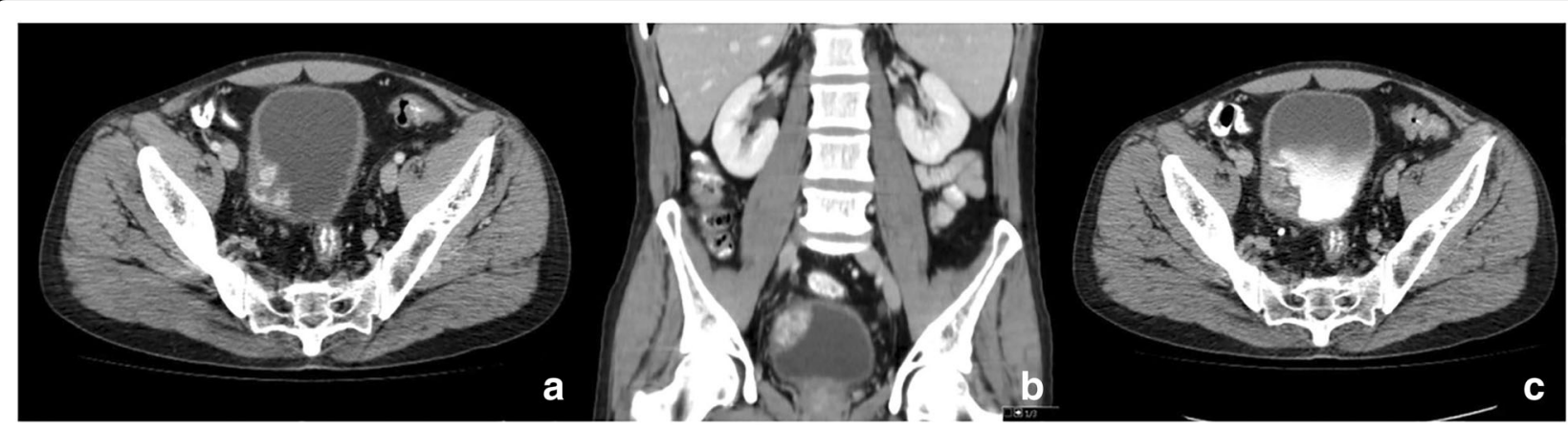

Fig. 5 SFT of the bladder. Axial (a) and coronal (b) CT images showing a int raves i cal heterogeneous vascularized mass. In the late phase axial images (c) the lobular contour is more conspicuous

signal intensity on T1-weighted images and heterogeneous low signal intensity with flow voids on T2- weighted images.

Contrast enhancement is usually avid, with nonenhancing areas occurring in larger lesions, which can represent foci of tissue necrosis or cystic or myxoid degeneration [20].

\section{Extra-pleural SFT by site}

The extrapleural SFTs diagnosis is challenging due to the wide differential diagnosis and the lack of specificity of extremity SFTs imaging features [5]. One unifying important extrapleural SFTs differential diagnosis is fibromatosis. Imaging features of SFTs generally also overlap with fibrosarcoma, synovial sarcoma and fibrous histiocytoma [19].

The series of extra-pleural SFT will be presented along the different anatomical locations.

\section{Extra-pleural thoracic SFT}

Small SFT of the pleura typically demonstrates at CT imaging a homogeneous well-defined, lobular, soft-tissue mass, adjacent to the chest wall or within a fissure, showing an obtuse angle with the pleural surface. Larger lesions are typically heterogeneous and may not exhibit CT features suggestive of pleural tumors.

An intermediate to high spontaneous attenuation is generally the case on unenhanced CT studies, which is due to the high density of collagen structure and the abundant vascularity of these lesions.

The contrast enhancement is typically intense and heterogenous conferring a geographic pattern of necrosis, hemorrhage and cystic and myxoid degeneration.

MR usually shows heterogeneous signal intensity on $\mathrm{T} 1$ and T2 weighted images with avid contrast enhancement following gadolinium administration. Areas of low T2 signal and lack of invasion of adjacent structures are some helpful features in suggesting this tumor.

Thoracic extra-pleural SFT generally present similar CT and MR characteristics as their pleural counterpart with no obvious connection with the pleural surfaces (Figs. 1 and 2).

To be noted that intrapulmonary location is exceeding rare for extrapleural SFTs [5].

\section{SFT of abdominal and pelvis peritoneum}

Abdominal and pelvic SFT commonly arise from the peritoneum presenting clinical and imaging features

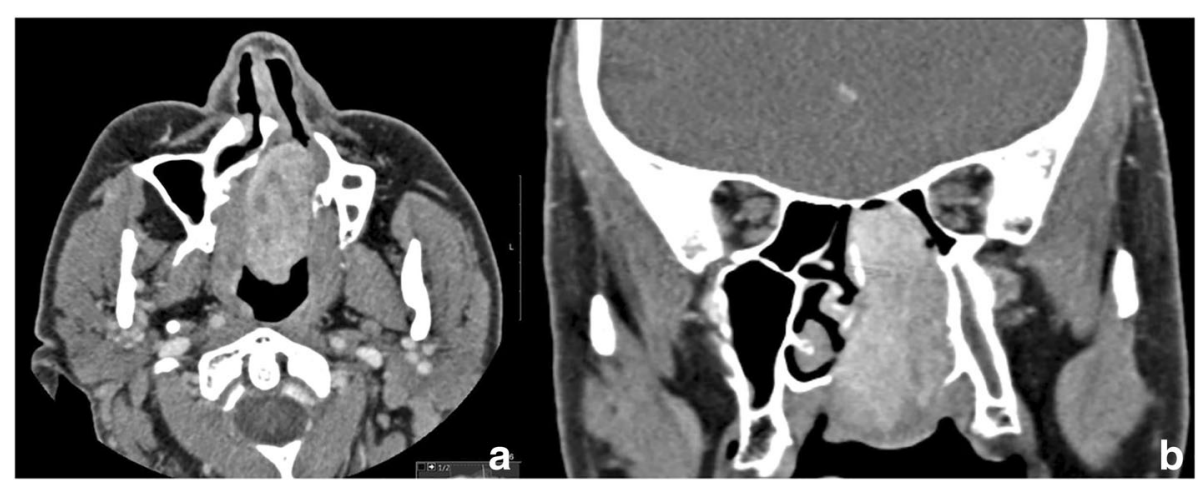

Fig. 6 SFT of the left nasal cavity. The left nasal cavity is filled with a solid, lobular, enhancing mass 


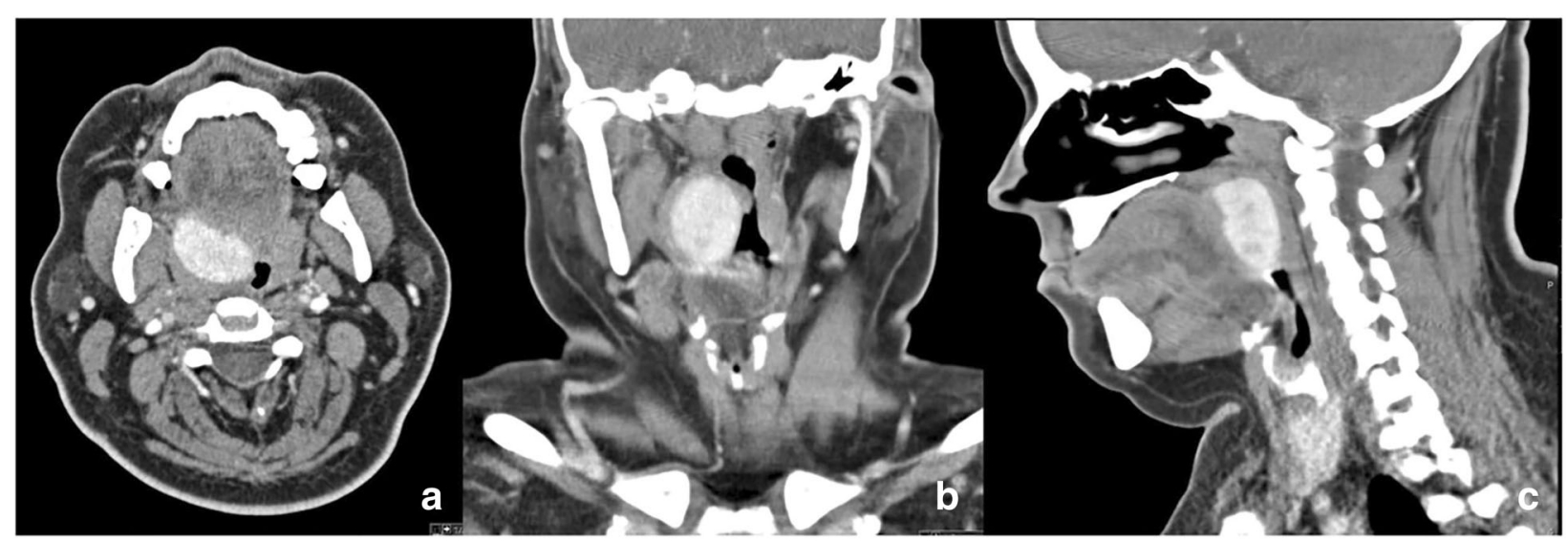

Fig. 7 SFT of the right palatine tonsil. Enhanced CT images (a-c) showing a well- defined homogeneous enhancing mass at the right palatine tonsil. No adenopathy is present

similar to those of the other extrathoracic location. They are more common in patients above 50 years old, often asymptomatic and large at presentation. Patients may experience symptoms associated to compression on contiguous organs, presence of a palpable mass, or hypoglycaemia [15]. Large pelvic SFTs have been reported to result in large bowel obstruction and various urinary symptoms including urinary retention and bilateral hydronephrosis [21].

Local recurrence and malignant degeneration has been reported in SFTs arising from the abdominal and pelvic peritoneum. SFTs of the peritoneum (Figs. 3 and 4) appear as hypervascular masses with intratumoral cystic changes, necrosis or hemorrhage like other extra-thoracic locations.
Low signal intensity with flow voids on T2-weighted images, representing fibrosis or collagen, are the most useful MR imaging features.

When arising in gastrointestinal system, SFT has to be essentially differentiated from gastrointestinal stromal tumors (GISTs).

Some pelvic organs such as prostate and the urinary bladder are very unusual locations for SFTs. Gross hematuria, urinary retention and pain are common symptoms. Regarding the bladder, a heterogeneous vascular tumor intravesicular or exophytic can be encountered (Fig. 5). The differential diagnosis in these locations comprises inflammatory pseudotumor, leiomyosarcoma, sarcomatoid transitional cell carcinoma, and pheochromocytoma.

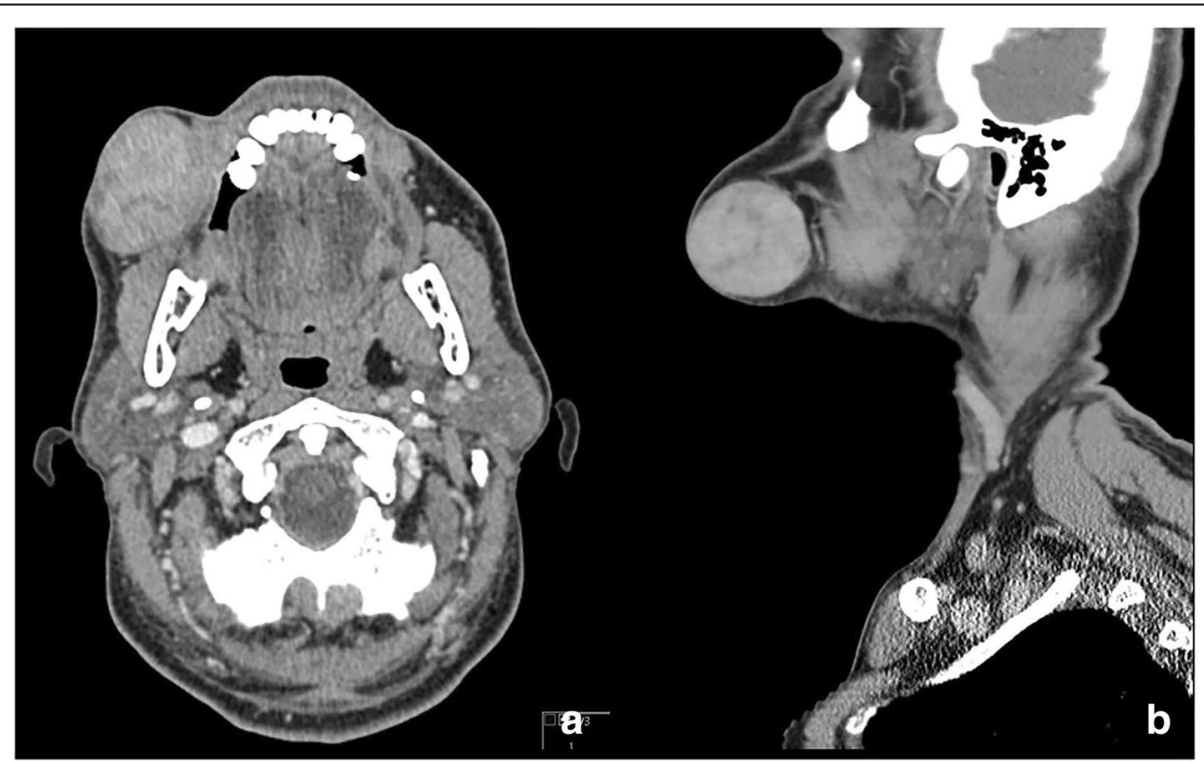

Fig. 8 SFT of the buccal space. Axial (a) and sagittal (b) CT images showing a spherical enhancing mass in the right buccal space, biopsy proven to be a SFT 


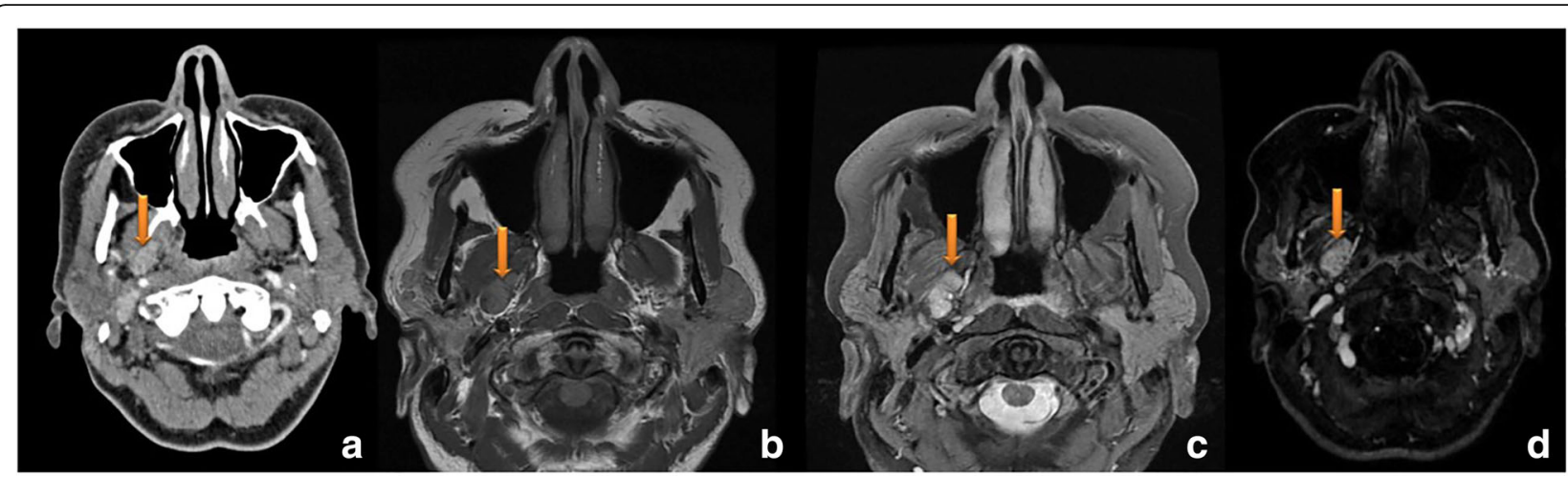

Fig. 9 SFT of the parapharyngeal space. Axial CT after administration of intravenous contrast medium demonstrates a small homogeneous soft tissue mass with lobular borders in the right parapharyngeal space. Axial T1-weighted (b), T2- weighted (c) and gadolinium-enhanced T1-

weighted MR images (d) showing a well-defined lesion hypointense on T1-weighted images, slightly hyperintense on T2-weighted images and avidly homogeneously enhancing

\section{SFT of the head and neck}

The head and neck regions are the most frequent extrathoracic locations of SFTs. Small series report that they tend to be more likely benign than SFTs of other locations [22].

The nasal cavity and paranasal sinuses are frequently involved (Figs. 6 and 7). There are also reports of SFTs in the larynx, nasopharynx and buccal space (Figs. 8, 9 and 10).

Intracranial SFTs are usually of dural origin. Dural tails and hyperostosis of the overlying calvaria is generally observed. The main differential diagnosis are meningioma, neurogenic tumor and soft-tissue sarcoma.

CT and MR imaging generally show, as in other locations, a well-circumscribed tumor, strongly enchanting after contrast administration.

At MR imaging, the usual signal features are observed, with the highlight that a low-signal-intensity rim representing a pseudocapsule around the lesion may be seen on T2-weighted images.

\section{SFT of soft tissue and bones}

Somatic soft tissues involving various locations, mainly the extremities and the head, are the origin of $10 \%$ of SFTs [23] (Figs. 11, 12, and 13). Only a few reports have described SFT involving the extremities. Most occur in the proximal lower extremities.

Imaging features of extremity SFTs lack specificity and correlate with the variable histopathological appearances.

Extra-thoracic SFTs generally show lobulated well defined margins, tending to displace adjacent structures. They also tend to be highly vascular with avid contrast enhancement.

Although rare, we should raise suspicion of a soft tissue SFT when a deep heterogeneous-mixed soft tissue mass with marked enhancement is observed.

Either benign and malignant tumors like neurogenic tumor, desmoid tumor, fibrosarcoma, synovial sarcoma and malignant fibrous histiocytoma may present overlap imaging and clinical features with SFTs.

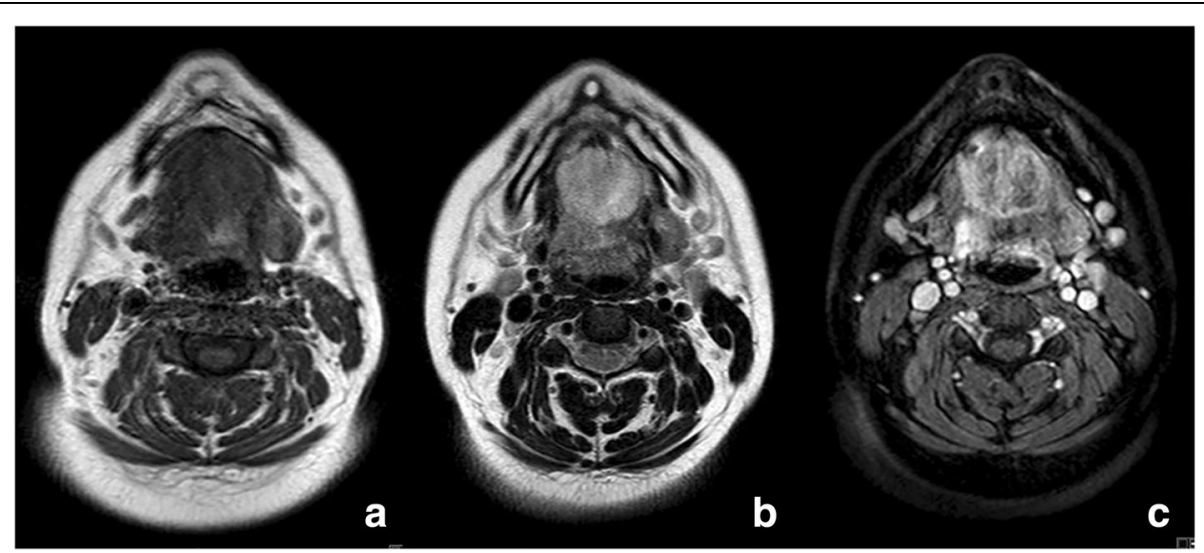

Fig. 10 SFT arising from the floor of the mouth. Axial T1-weighted (a), T2-weighted (b), and gadolinium-enhanced T1- weighted (c) MR images showing a large, well-defined mass. The mass has intermediate intensity signal on the T2-weighted image, isointense to muscle on the T1weighted image showing moderate heterogeneous enhancement on the gadolinium-enhanced images 


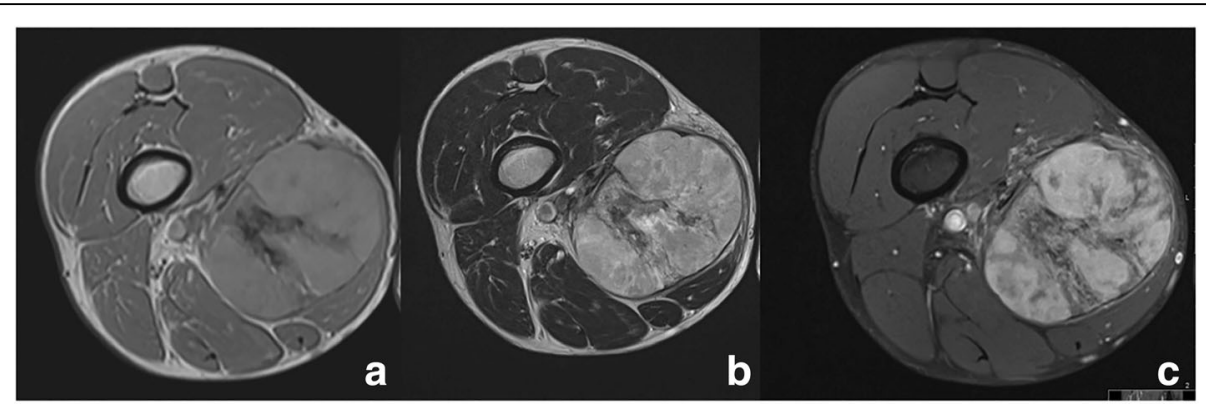

Fig. 11 Axial T1-weighted (a) T2-weighted (b) and contrast- enhanced MR images show a welldefined, enhancing soft-tissue mass in the medial compartment of the right thigh. Feeding vessels have low signal intensity in all sequences due to flow void

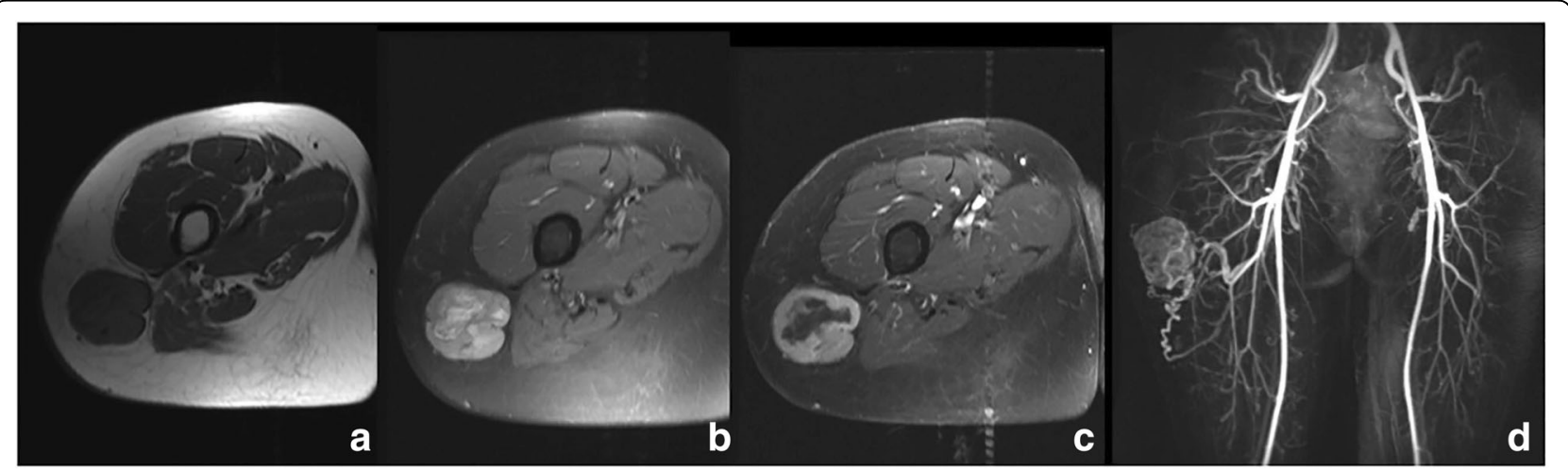

Fig. 12 Axial T1-weighted (a) T2-weighted fat saturated (b) and contrast-enhanced MR images (c) show well-defined, heterogeneously enhancing softtissue mass in the lateral compartment of the thigh. TOF sequence MR images (d) show the hypervascularity of the lesion, biopsy proven to be a SFT

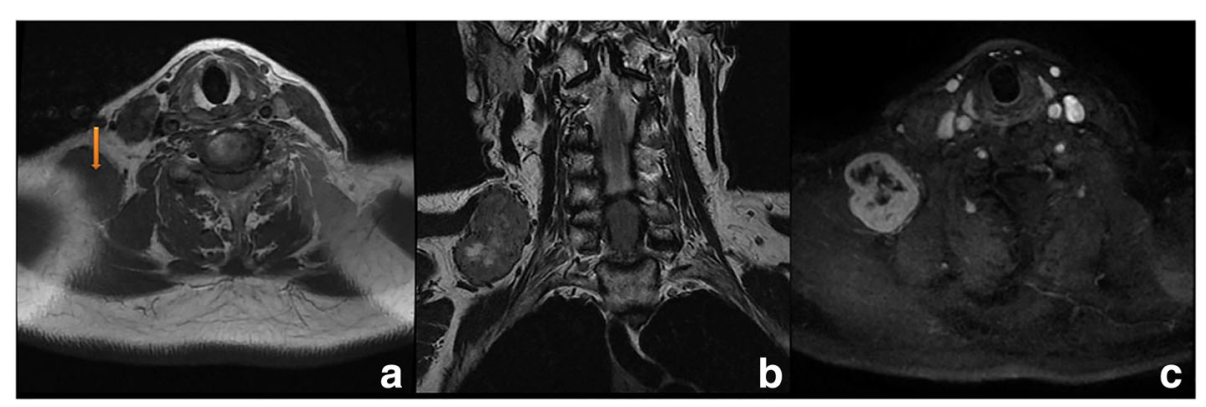

Fig. 13 Somatic SFT of the right supraclavicular fossa. MR images showing a large, well-defined enhancing mass at the right supraclavicular fossa. The tumor demonstrates signal isointensity relative to muscle on T1-weighted images (a), slight hiperintensity on T2-weighted images (b) and avid enhancement, with some non enhancing areas due to tissue necrosis on the contrast-enhanced MR images (c) 


\section{Conclusion}

Extrapleural SFTs are nowadays more frequently reported than pleural SFTs and generally manifest as large, slow-growing soft-tissue neoplasms.

A high index of suspicion of SFT should be raised in a deep heterogeneously enhancing soft tissue extrapleural mass in which MRI suggests fibrous content. A frequent and unifying feature of SFT at MR imaging is the presence of low signal-intensity foci on T1- and T2-weighted images, corresponding to the collagen content. Smaller tumors tend to enhance homogeneously, whereas larger lesions may have areas of myxoid or necrotic change. When a central focus of heterogeneity and variable contrast enhancement is identified in CT or MR images, malignant degeneration should be considered.

Due to the wide biologic behavior of these tumors the prediction of recurrence and metastasis is limited.

\section{Acknowledgements}

This research did not receive any specific grant from funding agencies in the public, commercial, or not-for-profit sectors.

\section{Competing interests}

The authors declare that they have no competing interests.

Received: 20 June 2018 Accepted: 4 September 2018

Published online: 16 October 2019

\section{References}

1. Gold JS, Antonescu CR, Hajdu C et al (2002) Clinico- pathologic correlates of solitary brous tumors. Cancer 94(4):1057-1068

2. Klemperer P, Rabin CB (1931) Primary neoplasms of the pleura: a report of five cases. Arch Pathol 11:385-412

3. Genger C, Guillou L (2006) Solitary fibrous tumor and hemangiopericytoma: evolution of a concept. Histopathology 48:63-74

4. Morimitsu Y, Nakajima M, Hisaoka M, Hashimoto H (2000) Extrapleural solitary fibrous tumor: clinicopathologic study of 17 cases and molecular analysis of the p53 pathway. APMIS 108(9):617-625

5. Ronchi A et al (2018) Extrapleural solitary fibrous tumor: a distinct entity from pleural solitary fibrous tumor. An update on clinical, molecular and diagnostic features. Ann Diagn Pathol 9(34):142-150

6. Levy AD, Rimola J, Mehrotra AK, Sobin LH (2006) From the archives of the AFIP: benign fibrous tumors and tumorlike lesions of the mesentery: radiologic-pathologic correlation. Radiographics 26(1):245-264

7. Cardinale L, Ardissone F, Garetto I, Marci V, Volpicelli G, Solitro F et al (2010) Imaging of benign solitary fibrous tumor of the pleura (SFTP): a pictorial essay. Rare Tumors 2:e1

8. Tam ES, Chen EC, Nijhawan N, Harvey JT, Howarth D, Oestreicher JH (2008) Solitary fibrous tumor of the orbit: a case series. Orbit 27:426-431

9. Shanbhogue A, Prasad S, Takahashi N, Vikram R, Zaheer A, Sandrasegaran K (2011) Somatic and visceral solitary fibrous tumors in the abdomen and pelvis: cross-sectional imaging spectrum. RadioGraphics 31:393-408

10. Wolfgang et al (2017) Solitary fibrous tumor: a center's experience and an overview of the symptomatology, the diagnostic and therapeutic procedures of this rare tumor. Resp Med Case Rep 21

11. Fletcher CDM, Bridge JA, Hogendoorn P, Mertens FWHO (2013) Classification of Tumours of Soft Tissue and Bone. IARC, Lyon, p 4

12. Espat NJ, Lewis JJ, Leung D et al (2002) Conventional hemangiopericytoma: modern analysis of outcome. Cancer 95(8):1746-1751

13. Cranshaw IM, Gikas A, Fisher C, Thway K, Thomas JM, Hayes AJ (2009) Clinical outcomes of extra-thoracic solitary fibrous tumours. Eur J Surg Oncol 35:994-998. https://doi.org/10.1016/j.ejso.2009.02.015

14. Peixoto Callejo I (2009) Peritoneal solitary fibrous tumour (SFT): long-term survival of recurrent and metastasised SFT treated with cytoreductive surgery and intraperitoneal chemotherapy. Clin Transl Oncol 11:250-252
15. Constantinidou A, Jones RL, Olmos D, Thway K, Fisher C, Al-Muderis O et al (2012) Conventional anthracycline-based chemotherapy has limited efficacy in solitary fibrous tumour. Acta Oncol 51:550-554

16. Park MS, Araujo DM (2009) New insights into the hemangiopericytoma/ solitary brous tumor spectrum of tumors. Curr Opin Oncol 21(4):327-331

17. Kawamura S, Nakamura T, Oya T et al (2007) Advanced malignant solitary brous tumor in pelvis responding to radiation therapy. Pathol Int 57(4):213-218

18. Francis M, Ayoub N, Thomas P (2012) Solitary fibrous tumor: an update on the spectrum of extrapleural manifestations. Skeletal Radiol 41:5-13

19. Krishnakumar S, Subramanian N, Mohan ER, Mahesh L, Biswas J, Rao NA (2003) Solitary fibrous tumor of the orbit: a clinicopathologic study of six cases with review of the literature. Surv Ophthalmol 48:544-554

20. Rosado-de-Christenson ML, Abbott GF, McAdams HP, Franks TJ, Galvin JR (2003) Localized fibrous tumors of the pleura. RadioGraphics 23(3):759-783

21. Yi B, Bewtra C, Yussef K, Silva E (2007) Giant pelvic solitary fibrous tumor obstructing intestinal and urinary tract: a case report and literature review. Am Surg 73:478-480

22. Ahmed R, Benjamin H (2011) Soft tissue tumors of the head and neck: imagingbased review of the WHO classification. RadioGraphics 31:1923-1954

23. Oliver W, Eleanor M, Khin T, Joseph T (2010) Solitary fibrous tumors of the soft tissues: review of the imaging and clinical features with histopathologic correlation. AJR 195:W55-W62

\section{Publisher's Note}

Springer Nature remains neutral with regard to jurisdictional claims in published maps and institutional affiliations.

\section{Submit your manuscript to a SpringerOpen ${ }^{\circ}$ journal and benefit from:}

- Convenient online submission

- Rigorous peer review

- Open access: articles freely available online

High visibility within the field

- Retaining the copyright to your article

Submit your next manuscript at $>$ springeropen.com 